\title{
Morning Blood Pressure is Associated with Sleep Quality in Obese Adolescents
}

\author{
Tamara S. Hannon, M.D., M.S. ${ }^{1}$, Wanzhu Tu, Ph.D. ${ }^{2}$, Sara E. Watson, M.D. ${ }^{1}$, Hasnaa Jalou, \\ M.D. ${ }^{1}$, Sangeeta Chakravorty, M.D. ${ }^{3}$, and Silva Arslanian, M.D. ${ }^{3}$ \\ ${ }^{1}$ Department of Pediatrics, Indiana University School of Medicine, Indianapolis, IN 46022 \\ ${ }^{2}$ Biostatistics, Indiana University School of Medicine, Indianapolis, IN 46022 \\ ${ }^{3}$ Department of Pediatrics, Children's Hospital of Pittsburgh, University of Pittsburgh Medical \\ Center, Pittsburgh, PA 15224
}

\begin{abstract}
Objective-To examine relationships between blood pressure (BP), adiposity, and sleep quality using overnight polysomnography (PSG) in obese adolescents.

Study design-Overnight PSG and morning BP measurements were performed in obese (BMI $>97$ th \%ile) non-diabetic adolescents (eligible age range $12-18$ years, $n=49$ ). Subjects were stratified into two groups, one with normal BP, and one with elevated BP, and demographic and clinical characteristics compared between the groups. Multiple linear regression analysis was used to assess the BP effects of sleep quality measures.
\end{abstract}

Results-Participants ( $\mathrm{n}=27$ ) had normal morning BP, and 22 (44.9\%) had elevated morning BP. There were no differences in age $(\mathrm{p}=0.53)$, sex $(\mathrm{p}=0.44)$, race $(\mathrm{p}=0.58)$ or $\mathrm{BMI}(\mathrm{p}=0.56)$ between the two BP groups. The group with elevated BP spent shorter percentages of time in rapid eye movement (REM; $p=0.006$ ) and slow-wave sleep (SWS; $\mathrm{p}=0.024)$. Multiple linear regression analysis showed a lower percent of both REM and SWS were associated with increased morning $\mathrm{BP}$, after adjusting for pubertal stage, sex, race, and BMI.

Conclusion-Lack of deeper stages of sleep, REM sleep and SWS, is associated with higher morning BP in obese adolescents, independent of BMI. Poor sleep quality should be considered in the work-up of obese youth with hypertension. Intervention studies are needed to evaluate whether improving the quality of sleep will reduce blood pressure elevation.

\section{Keywords}

obesity; hypertension; sleep disorders; obstructive sleep apnea; overweight

Increasing prevalence of both hypertension and obesity-related sleep disorders in youth are directly associated with the obesity epidemic $(1,2)$. Studies in adults have shown that poor sleep quality and hypertension are linked, and this relationship is independent of obesity (3-7). Moreover, the association between BP and sleep quality in adults is characterized by

\footnotetext{
(C) 2013 Mosby, Inc. All rights reserved

Corresponding Author and Contact Information: Tamara S. Hannon, MD, MS 705 Riley Hospital Drive, Room 5960 Indianapolis, IN 46202-5225 Office: 317-944-3889 Fax: 317-944-3882 tshannon@iu.edu.

Publisher's Disclaimer: This is a PDF file of an unedited manuscript that has been accepted for publication. As a service to our customers we are providing this early version of the manuscript. The manuscript will undergo copyediting, typesetting, and review of the resulting proof before it is published in its final citable form. Please note that during the production process errors may be discovered which could affect the content, and all legal disclaimers that apply to the journal pertain.
} 
sleep fragmentation and a relative lack of restorative deeper sleep, particularly slow-wave sleep (SWS), during which sympathetic nervous system activity decreases and heart rate and blood pressure drop $(8,9)$.

In adolescents, whether or not there is an additional impact of poor sleep quality on BP above and beyond that associated with by obesity is not understood. The objective of this study was to examine relationships between adiposity, BP, and objective measures of sleep quality measured by polysomnogram (PSG). We performed a secondary analysis of a study designed to evaluate insulin sensitivity and measures of sleep quality in obese adolescents, in which morning BP measures were also collected (10). The primary hypothesis was that morning BP would be higher among patients with poorer sleep quality characterized by less deep sleep (SWS), independent of body mass index (BMI).

\section{Methods}

The study was approved by the University of Pittsburgh and Indiana University Institutional Review Boards and performed in the Pediatric Clinical and Translational Research Centers at these institutions. This was a secondary analysis of a study powered to detect the association between insulin sensitivity and sleep disordered breathing measured using the apnea-hypopnea index (AHI) as the primary outcome in obese black and white adolescents (10). Data from this secondary analysis have not been previously reported.

Non-diabetic patients referred for clinical evaluation of obesity who met study criteria were invited to participate in the original study. Participants were not previously identified as having hypertension or sleep disorders. After obtaining informed assent/consent, we studied 50 obese (BMI >97th \%ile) non-diabetic adolescents (eligible age range 12-18 years). Exclusion criteria included airway disease, smoking, uncontrolled chronic disease or current use of chronic medications affecting glucose regulation or BP, and obesity associated with a syndrome, such as Prader-Willi syndrome.

Overnight PSG was performed and data were recorded using either Sensormedics Somnostar Pro version 7.2 software or Sandman Elite 9.1 sleep diagnostic software, applying the following EEG montage: F3M2, F4M1, C3M2, C4M1, O2M1, O1M2, L-EOG, R-EOG, chin EMG, limb EMG, and the following cardiorespiratory measurements: $\mathrm{SpO} 2$ and pulse (Masimo), nasal pressure, airflow (nasal or oral thermistor), thoracic and abdominal excursion (uncalibrated respirator inductance plethysmography), pulse and ECG. The PSG data were interpreted by one of two sleep medicine co-investigators. The apnea-hypopnea index (AHI), which is the total number of obstructive apnea and hypopnea events per one hour of sleep, was hand-scored utilizing pediatric criteria and calculated following the AASM manual for scoring guidelines (11). One participant's data were excluded from the analysis because very severe obstructive sleep apnea was apparent on the PSG (AHI = 50 events per hour).

Morning BP following the PSG (0600-0700) was measured in duplicate (at 10 minute intervals) by a trained research nurse with a calibrated automated oscillometric device (Dynamap) using a cuff appropriate for arm size with the participant awake, seated and at rest. The analysis was based on the average morning systolic (SBP) and diastolic BP (DBP). Elevated BP was identified if the SBP or DBP was either at least $\geq 90^{\text {th }}$ percentile for age, sex, and height, if the SBP exceeded $120 \mathrm{mmHg}$ or the DBP exceeded $80 \mathrm{mmHg}$ (12).

Percent body fat was measured by dual-energy X-ray absorptiometry (DEXA) in all but 4 participants. In the remaining participants, DEXA was not performed due to the participant's weight exceeding the limits for the equipment. Pubertal stage was determined by physical examination (13). 


\section{Statistical Analyses}

Study subjects were stratified into two groups, one with normal BP, and one with elevated BP (as defined above). Demographic and clinical characteristics of the subjects were summarized and compared between the groups. Categorical variables were compared using the chi-square test, and continuous variables were compared using the two-sample $t$ test and a nonparametric test (Mann-Whitney U statistic) was used for non-normally distributed measures. Scatter plots were used to graphically examine the relationships between sleep characteristics obtained from the PSG, body mass index (BMI), waist circumference, and BP. Pearson's correlation coefficients were used to quantify the magnitudes of the linear correlations. Multiple linear regression analysis was then conducted to assess the BP effects of sleep quality measures, including the percent of sleep time spent in rapid eye movement (REM) sleep and slow-wave sleep (SWS), and the AHI, while controlling for the effects of age or pubertal stage, sex, race (as a dichotomous variable), and BMI. All analyses were implemented using SPSS software (Version 20). P values $<0.05$ were considered statistically significant.

\section{Results}

The characteristics of the study participants are reported in Table I. The study included 49 participants; mean age $14.4 \pm 1.7$ (SD) years; $51.9 \%$ male; $57.1 \%$ white, $40.7 \%$ black, and $2.0 \%$ mixed-race (black/white). Participants $(n=27)$ had morning BP values in the normal range, and $22(44.9 \%)$ had elevated morning BP. There were no significant differences in age, pubertal stage (Tanner 4 in the normal BP group versus 5 in the high BP group, $\mathrm{p}=0.114$ ), sex, race or measures of adiposity between the two BP groups.

The group with elevated BP spent significantly shorter percentages of sleep time in REM sleep and SWS compared with the group with BP in the normal range (Table I). There were no significant differences in PSG sleep time, sleep latency, time to REM sleep, or indices of sleep disordered breathing including AHI, arousal index, and minimum $\mathrm{SaO}_{2}$ (Table I).

Morning DBP was positively associate with percent body fat ( $\mathrm{r}=0.33, \mathrm{p}=0.03)$; however, other measures of adiposity (BMI, BMI SDS, waist circumference) were not significantly associated with BP. Both SBP and DBP were inversely related to the percent of sleep time spent in REM and SWS sleep stages (Figure). The strongest relationship was between SBP and the percent of sleep time spent in REM sleep. The AHI was not associated with morning SBP $(r=0.094, p=0.52)$ or DBP $(r=0.030, p=0.84)$. We observed significant negative associations between the minimum percent $\mathrm{SaO}_{2}$ and waist circumference $(\mathrm{r}=-0.36, \mathrm{p}=0.01)$, and BMI SDS ( $\mathrm{r}=-0.34, \mathrm{p}=0.02)$, indicating an association between central adiposity and sleep disordered breathing.

Multiple linear regression analysis showed a lower percent of sleep time spent in both REM sleep and SWS were associated with significantly increased morning SBP and DBP, after adjusting for the effects of either pubertal stage, sex, race, and BMI (Tables II and III). The AHI, pubertal stage, sex, race, and BMI did not contribute significantly to the models. Specifically, for each $10 \%$ decrease in REM sleep, SBP on average increased by $3.9 \mathrm{mmHg}$ $(\mathrm{p}=0.011)$ and DBP on average increased by $3.0 \mathrm{mmHg}$. For each $10 \%$ decrease in SWS, DBP increased by $3.9 \mathrm{mmHg}$.

\section{Discussion}

In this study of obese adolescent research volunteers, we found that the percent of time spent in REM sleep was significantly inversely correlated with morning SBP and DBP, and the percent of time spent in SWS was significantly inversely correlated with morning DBP. 
These associations between REM and SWS sleep time and BP were not modified when accounting for pubertal stage, race, sex, and BMI. These results support the primary hypothesis that morning BP is higher among obese adolescents with poorer sleep quality characterized by less time spent in deep sleep stages, regardless of BMI. These results are also congruent with mounting evidence in the literature on adults that sleep disruption with reduction in deeper stages of sleep contributes to hypertension. Previous studies show that both acute and chronic sleep deprivation raise BP in adult volunteers (14). Longitudinal studies in older adults show that decreased SWS is associated with increased risk of developing hypertension $(13,14)$. Furthermore, there is an inverse association between BP and REM sleep among adults with drug-resistant hypertension (15).

There is a relative lack of pediatric studies evaluating relationships between sleep and BP, especially in obese adolescents. Our data are in agreement with the adult studies showing that lack of REM sleep is associated with higher morning SBP and DBP. We also observed an association between SWS and DBP in our study, consistent with studies of adults. A previous study in healthy adolescents found that short sleep duration and low sleep efficiency were both related to higher SBP and DBP, and these associations were not explained by socioeconomic status, obesity, or sleep disordering breathing $(16,17)$. However, the previous study found no specific association between PSG measured time spent in REM sleep or SWS (16). The children in the previous study had lower BMIs (mean BMI percentile 72.2, range 47.1-93.8) compared with the participants in our study who all had BMIs above the $95^{\text {th }}$ percentile (16). It is possible that our study participants were physiologically more similar to adults due to the severity of their obesity. Also, we did not see a significant relationship between SWS and SBP in our study. This may be at least partially explained by the small sample size and insufficient power of the secondary analysis to detect this.

In addition to being obese, the participants in our study had evidence of mild sleep disordered breathing (AHI $\geq 1.5$ ). Adult sleep disordered breathing with obstructive sleep apnea is associated with poor sleep quality, less REM sleep, less SWS, less physiologic nocturnal BP dipping, and higher morning and daytime BP $(14,18)$. Moreover, physiologic nocturnal BP dipping is significantly lessened when there is a deficiency of SWS, and this phenomenon is more apparent in older versus younger individuals $(18,19)$. Children with sleep disordered breathing also have higher daytime and nocturnal BP (20-22). However, in our study, we did not find an association between morning BP and measures of sleep disordered breathing ( $\mathrm{AHI}$ and $\mathrm{SaO}_{2}$ ). This is not altogether surprising, because our participants' degree of sleep disordered breathing was mild overall and didn't include a wide range of AHI values. We suspect that there was too little variation in the participants' AHI values to detect a significant association between sleep disordered breathing and morning BP in this study.

The effect of adiposity on BP intensifies as children become obese (23). The BP-adiposity relationship is modulated by a combination of factors, and BP measures are more strongly associated with adiposity indices among healthy populations with a range of degrees of body fatness (24). In the current study, morning DBP was, indeed, positively associated with percent body fat, and SBP was not associated with measures of adiposity. We propose that this is because the study participants were all obese and the study was originally powered to measure insulin sensitivity, with BP being a secondary outcome. Similarly, we did not observe an association between the degree of obesity and measures of sleep quality (sleep duration or percent of time spent in REM and SWS) in this study. It is possible that when a high level of adiposity is reached, the relationships between the degree of obesity and measures of BP and sleep quality are no longer linear. Moreover, although obesity may be the predisposing risk factor for these conditions, there are several other risk factors 
influencing BP and sleep quality measures, including genetics and lifestyle factors that could not be accounted for in this study.

Previously proposed mechanistic pathways linking poor sleep quality to adverse BP outcomes include a lack of nocturnal BP dipping, increased sympathetic nervous system activity, activation of the hypothalamic-pituitary-adrenal axis, and alterations in the reninangiotensin system (8). Although our study was not designed to evaluate the mechanisms of this relationship, it indicates the need for further studies examining such factors in the obese adolescent population, as advances in the understanding of the pathophysiology of adolescent sleep deficits and BP may have important public health implications with regard to policies to optimize sleep in children.

This study has limitations, including the cross-sectional design, a small sample size and lack of more extensive ambulatory BP measures. Additionally, the study population was restricted an obese adolescent referral population, potentially biasing the sample toward higher BP. We utilized automated BP measurements, which can overestimate SBP. This would have biased the results toward non-significance, and thus was not likely to change the findings. We also didn't measure BP during sleep. Additional limitations of our study are the exclusion of other race groups and the lack of information on socioeconomic status and family environment. Race has been shown to be a factor in BP during sleep in youth, with blacks having higher BP than whites (25). We did not observe a race-effect on BP, and this could be due to small sample size, referral bias, or it could be that there was an upper-limit obesity effect that overrode the race effect. Data on socioeconomic status, family environment, and race will be critical to include in future investigations of sleep effects on BP.

Evaluation of the obese adolescent should include careful assessment of both BP and signs and symptoms associated with poor sleep quality, including snoring and daytime sleepiness.

Moreover, poor sleep quality should be considered in the work-up of obese youth with hypertension. From a research perspective, these relationships should be investigated further to determine whether or not optimization of sleep along with traditional lifestyle change recommendations for diet and physical activity would improve BP in obese adolescents.

\section{Acknowledgments}

Supported by the National Institutes of Health (R03HD057532 to T.H.), Indiana University Purdue University

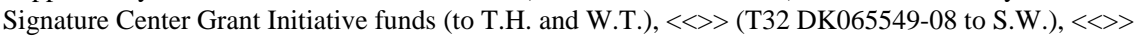
(K12DK063704 to S.A.), 〈〈〉> (UL1RR024153-CTSA), and Indiana CTSI CRC (TR000006). The authors declare no conflicts of interest.

\section{References}

1. Falkner B. Hypertension in children and adolescents: epidemiology and natural history. Pediatr Nephrol. 2010; 25:1219-24. [PubMed: 19421783]

2. Hart CN, Cairns A, Jelalian E. Sleep and obesity in children and adolescents. Pediatric Clinics of North America. 2011; 58:715-33. [PubMed: 21600351]

3. Bansil P, Kuklina EV, Merritt RK, Yoon PW. Associations between sleep disorders, sleep duration, quality of sleep, and hypertension: results from the National Health and Nutrition Examination Survey, 2005 to 2008. J Clin Hypertens. 2011; 13:739-43.

4. Fernandez-Mendoza J, Vgontzas AN, Liao D, Shaffer ML, Vela-Bueno A, Basta M, et al. Insomnia with objective short sleep duration and incident hypertension: the Penn State Cohort. Hypertension. 2012; 60:929-35. [PubMed: 22892811] 
5. Gangwisch JE, Feskanich D, Malaspina D, Shen S, Forman JP. Sleep Duration and Risk for Hypertension in Women: Results from The Nurses' Health Study. American Journal of Hypertension. 2013; 26:903-11. [PubMed: 23564028]

6. Zou D, Eder DN, Eskandari D, Grote L, Bostrom KB, Lindblad U, et al. Association between short total sleep time and hypertension: the Skara Sleep Cohort. Journal of Hypertension. 2013; 31:34551. [PubMed: 23196899]

7. Knutson KL, Van Cauter E, Rathouz PJ, Yan LL, Hulley SB, Liu K, et al. Association between sleep and blood pressure in midlife: the CARDIA sleep study. Archives of Internal Medicine. 2009; 169:1055-61. [PubMed: 19506175]

8. Javaheri S, Redline S. Sleep, slow-wave sleep, and blood pressure. Current Hypertension Reports. 2012; 14:442-8. [PubMed: 22846982]

9. Fung MM, Peters K, Redline S, Ziegler MG, Ancoli-Israel S, Barrett-Connor E, et al. Decreased slow wave sleep increases risk of developing hypertension in elderly men. Hypertension. 2011; 58:596-603. [PubMed: 21876072]

10. Hannon TS, Lee S, Chakravorty S, Lin Y, Arslanian SA. Sleep-disordered breathing in obese adolescents is associated with visceral adiposity and markers of insulin resistance. Pediatric Obesity. 2011; 6:157-60.

11. Iber, C. American Academy of Sleep Medicine. American Academy of Sleep Medicine; Westchester, IL: 2007. The AASM manual for the scoring of sleep and associated events : rules, terminology, and technical specifications; p. 59

12. The fourth report on the diagnosis, evaluation, and treatment of high blood pressure in children and adolescents. Pediatrics. 2004; 114:555-76. [PubMed: 15286277]

13. Marshall WA, Tanner JM. Growth and physiological development during adolescence. Annual Review of Medicine. 1968; 19:283-300.

14. Van Cauter E, Spiegel K, Tasali E, Leproult R. Metabolic consequences of sleep and sleep loss. Sleep Medicine. 2008; 9:S23-8. [PubMed: 18929315]

15. Friedman O, Bradley TD, Ruttanaumpawan P, Logan AG. Independent association of drugresistant hypertension to reduced sleep duration and efficiency. American Journal of Hypertension. 2010; 23:174-9. [PubMed: 19927130]

16. Javaheri S, Storfer-Isser A, Rosen CL, Redline S. Sleep quality and elevated blood pressure in adolescents. Circulation. 2008; 118:1034-40. [PubMed: 18711015]

17. Archbold KH, Vasquez MM, Goodwin JL, Quan SF. Effects of sleep patterns and obesity on increases in blood pressure in a 5-year period: report from the Tucson Children's Assessment of Sleep Apnea Study. The Journal of Pediatrics. 2012; 161:26-30. [PubMed: 22284918]

18. Sayk F, Teckentrup C, Becker C, Heutling D, Wellhoner P, Lehnert H, et al. Effects of selective slow-wave sleep deprivation on nocturnal blood pressure dipping and daytime blood pressure regulation. American Journal of Physiology Regulatory, Integrative and Comparative Physiology. 2010; 298:R191-7.

19. Sasaki N, Ozono R, Yamauchi R, Teramen K, Munemori M, Hamada H, et al. Age-related differences in the mechanism of nondipping among patients with obstructive sleep apnea syndrome. Clin Exp Hypertens. 2012; 34:270-7. [PubMed: 22559060]

20. Horne RS, Yang JS, Walter LM, Richardson HL, O'Driscoll DM, Foster AM, et al. Elevated blood pressure during sleep and wake in children with sleep-disordered breathing. Pediatrics. 2011; 128:e85-92. [PubMed: 21708802]

21. Amin R, Somers VK, McConnell K, Willging P, Myer C, Sherman M, et al. Activity-adjusted 24hour ambulatory blood pressure and cardiac remodeling in children with sleep disordered breathing. Hypertension. 2008; 51:84-91. [PubMed: 18071053]

22. Amin RS, Carroll JL, Jeffries JL, Grone C, Bean JA, Chini B, et al. Twenty-four-hour ambulatory blood pressure in children with sleep-disordered breathing. American journal of respiratory and critical care medicine. 2004; 169:950-6. [PubMed: 14764433]

23. Tu W, Eckert GJ, DiMeglio LA, Yu Z, Jung J, Pratt JH. Intensified effect of adiposity on blood pressure in overweight and obese children. Hypertension. 2011; 58:818-24. [PubMed: 21968752] 
24. Kotchen TA, Grim CE, Kotchen JM, Krishnaswami S, Yang H, Hoffmann RG, et al. Altered relationship of blood pressure to adiposity in hypertension. American journal of hypertension. 2008; 21:284-9. [PubMed: 18311125]

25. Harshfield GA, Alpert BS, Pulliam DA, Somes GW, Wilson DK. Ambulatory blood pressure recordings in children and adolescents. Pediatrics. 1994; 94:180-4. [PubMed: 8036070] 

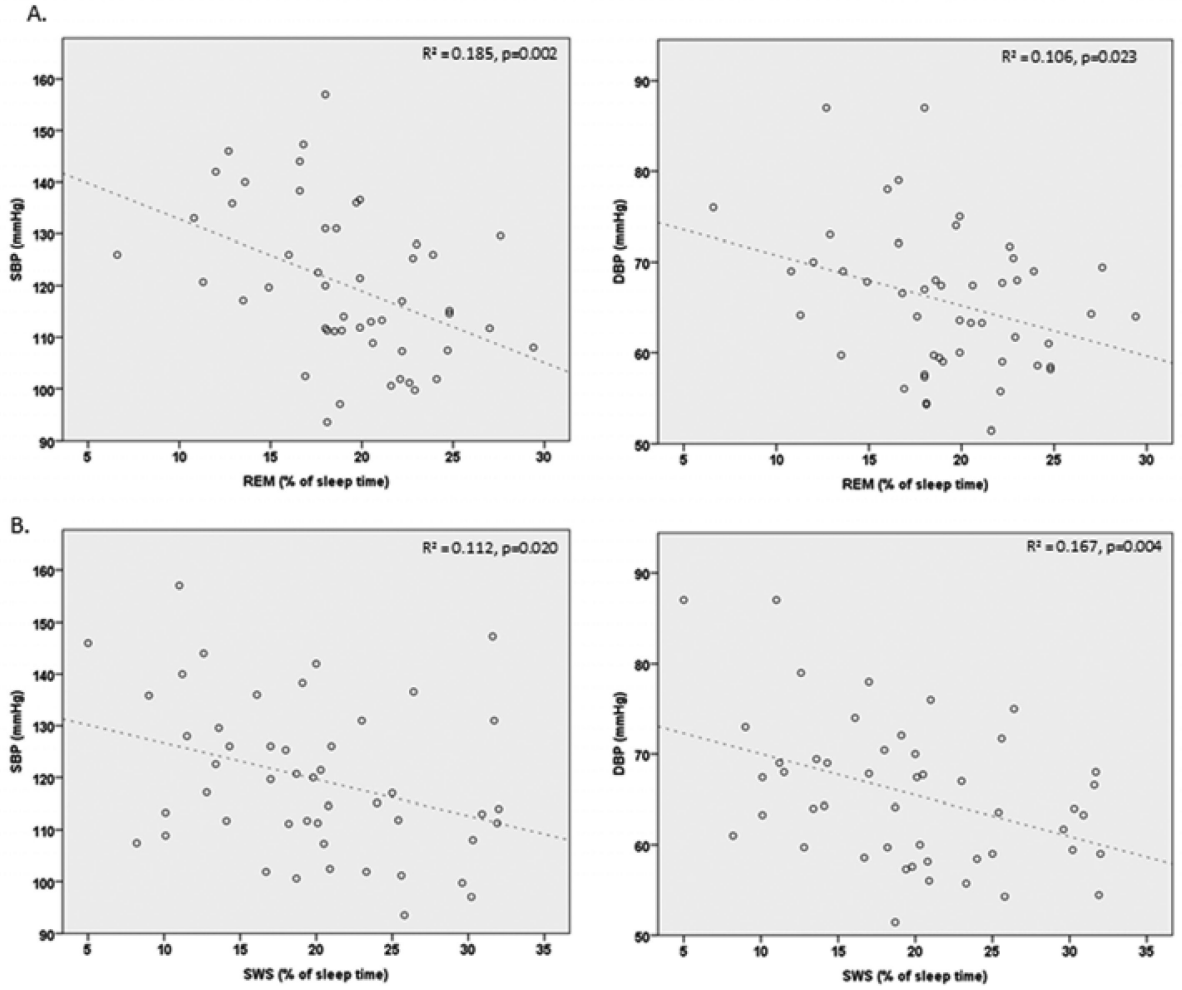

Figure.

Linear relationships between systolic and diastolic blood pressure with percent of time spent in A, rapid eye movement and B, slow wave sleep stages. 
Table 1

Characteristics of the study participants

\begin{tabular}{|c|c|c|c|}
\hline & $\begin{array}{c}\text { BP normal } \\
\qquad \begin{array}{c}n \\
=27\end{array}\end{array}$ & $\begin{array}{c}\text { BP elevated } \\
\quad n=22\end{array}$ & $P$ \\
\hline \multicolumn{4}{|l|}{ A.M. Blood Pressure } \\
\hline \multirow[t]{2}{*}{ SBP (mmHg) } & $109 \pm 7$ & $134 \pm 9$ & $<0.001$ \\
\hline & $(111 ; 94-120)$ & $(132 ; 121-157)$ & \\
\hline \multirow[t]{2}{*}{ DBP (mmHg) } & $61 \pm 5$ & $72 \pm 7$ & $<0.001$ \\
\hline & $(60 ; 51-72)$ & $(70 ; 60-87)$ & \\
\hline \multicolumn{4}{|l|}{ Demographics } \\
\hline Age (years) & $14.3 \pm 1.7$ & $14.6 \pm 1.6$ & 0.533 \\
\hline Sex & & & 0.445 \\
\hline Male, n (\%) & $14(51.9)$ & $9(40.9)$ & \\
\hline Female, n (\%) & $13(48.1)$ & $13(59.1)$ & \\
\hline Race & & & 0.585 \\
\hline White, $\mathrm{n}(\%)$ & $16(59.3)$ & $12(54.5)$ & \\
\hline Black, n (\%) & $10(37.0)$ & $10(45.5)$ & \\
\hline Mixed-race, n (\%) & $1(3.7)$ & $0(0.0)$ & \\
\hline \multicolumn{4}{|l|}{ Adiposity Measures } \\
\hline \multirow[t]{2}{*}{ BMI $\left(\mathrm{kg} / \mathrm{m}^{2}\right)$} & $38.7 \pm 8.9$ & $39.9 \pm 8.5$ & 0.560 \\
\hline & $(35.0 ; 28.4-61.6)$ & $(37.9 ; 28.7-57.1)$ & \\
\hline \multirow[t]{2}{*}{ BMI SDS } & $3.95 \pm 1.65$ & $3.92 \pm 1.41$ & 0.936 \\
\hline & $(3.55 ; 1.80-8.31)$ & $(3.50 ; 2.04-6.31)$ & \\
\hline Body fat (\%) & $46.2 \pm 4.62$ & $47.0 \pm 7.4$ & 0.674 \\
\hline$(\mathrm{n}=41)$ & $(46.4 ; 37.5-53.8)$ & $(46.3 ; 36.7-58.5)$ & \\
\hline \multirow[t]{2}{*}{ Waist (cm) } & $114.8 \pm 17.7$ & $114.3 \pm 19.6$ & 1.00 \\
\hline & $(108.2 ; 91.4-155.0)$ & $(112.2 ; 78.1-149.0)$ & \\
\hline \multicolumn{4}{|l|}{$\underline{\text { Sleep Measures }}$} \\
\hline \multirow[t]{2}{*}{ REM (\%) } & $20.9 \pm 3.6$ & $17.0 \pm 4.9$ & 0.003 \\
\hline & $(20.6 ; 13.5-29.4)$ & $(17.2 ; 6.6-27.6)$ & \\
\hline \multirow[t]{2}{*}{ SWS (\%) } & $21.5 \pm 6.9$ & $17.4 \pm 6.9$ & 0.043 \\
\hline & $(20.8 ; 8.2-32.0)$ & $(17.0 ; 5.0-31.7)$ & \\
\hline \multirow[t]{2}{*}{ PSG sleep time (minutes) } & $421.2 \pm 28.5$ & $396.7 \pm 75.2$ & 0.159 \\
\hline & $(417.0 ; 354.0-483.5)$ & $(394.7 ; 233.0-494.0)$ & \\
\hline \multirow[t]{2}{*}{ Sleep latency (minutes) } & $13.9 \pm 11.7$ & $18.3 \pm 16.4$ & 0.382 \\
\hline & $(12.0 ; 1.0-46.5)$ & $(15.2 ; 0.5-67.0)$ & \\
\hline \multirow[t]{2}{*}{ Time to REM (minutes) } & $118.8 \pm 44.3$ & $133.4 \pm 70.7$ & 0.819 \\
\hline & $(116.5 ; 50.0-221.5)$ & $(101.5 ; 42.0-276.0)$ & \\
\hline \multirow[t]{2}{*}{ AHI (events/hour) } & $2.9 \pm 2.9$ & $4.6 \pm 5.5$ & 0.880 \\
\hline & $(1.7 ; 0.1-12.4)$ & $(1.2 ; 0.0-20.0)$ & \\
\hline \multirow[t]{2}{*}{ Arousal index (events/hour) } & $9.2 \pm 4.8$ & $9.3 \pm 5.2$ & 0.976 \\
\hline & $(8.4 ; 4.1-25.3)$ & $(7.9 ; 2.0-22.2)$ & \\
\hline
\end{tabular}

J Pediatr. Author manuscript; available in PMC 2015 February 01. 


\begin{tabular}{cccc} 
& BP normal & BP elevated & $\boldsymbol{P}$ \\
$\mathbf{n = 2 7}$ & $\mathbf{n = 2 2}$ & \\
\hline Minimum $\mathrm{SaO}_{2}(\%)$ & $89.9 \pm 4.7$ & $89.3 \pm 5.3$ & 0.864 \\
& $(92 ; 77-95)$ & $(90.5 ; 76-96)$ & \\
\hline
\end{tabular}

Data are expressed as mean $\pm \mathrm{SD}$ (median; range) or mean (\%) 
Table 2

Multiple linear regression coefficients (standardized beta) for the associations between measures of sleep quality and A.M. SBP

\begin{tabular}{lrr}
\hline & Coefficient & p-value \\
\hline${ }^{*}$ REM (\%) & -0.387 & 0.011 \\
${ }^{*}$ SWS (\%) & -0.219 & 0.142 \\
${ }^{*}$ AHI & 0.057 & 0.707 \\
Sex & -0.069 & 0.759 \\
Race & 0.056 & 0.706 \\
Pubertal stage & 0.175 & 0.476 \\
BMI & 0.095 & 0.549 \\
\hline
\end{tabular}

REM and SWS (\%) and AHI (total) variables were entered into the model 


\section{Table 3}

Multiple linear regression coefficients (standardized beta) for the associations between measures of sleep quality and A.M. DBP

\begin{tabular}{lrr}
\hline & Coefficient & p-value \\
\hline${ }^{*}$ REM (\%) & -0.301 & 0.048 \\
${ }^{*}$ SWS (\%) & -0.390 & 0.013 \\
*AHI & -0.039 & 0.802 \\
Sex & -0.063 & 0.782 \\
Race & -0.052 & 0.728 \\
Age & -0.018 & 0.943 \\
BMI & 0.203 & 0.214 \\
\hline
\end{tabular}

REM and SWS (\%) and AHI (total) variables were entered into the model 